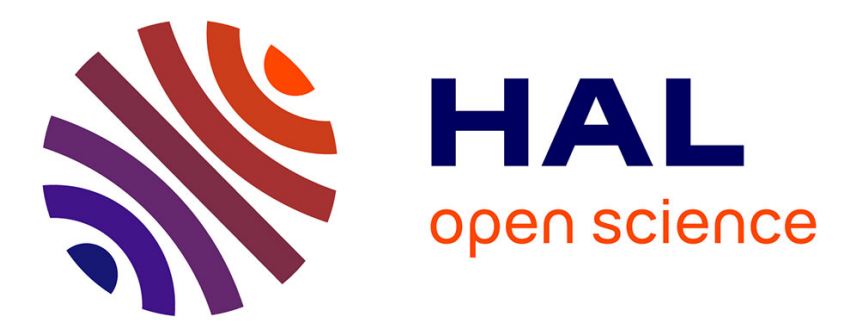

\title{
Magneto Elastic Modes in Ferro or Ferrimagnetic Polycristals
}

\author{
G. Le Blevennec, I. Brard
}

\section{To cite this version:}

G. Le Blevennec, I. Brard. Magneto Elastic Modes in Ferro or Ferrimagnetic Polycristals. Journal de Physique IV Proceedings, 1997, 07 (C1), pp.C1-191-C1-192. 10.1051/jp4:1997170 . jpa-00255117

\section{HAL Id: jpa-00255117 https://hal.science/jpa-00255117}

Submitted on 1 Jan 1997

HAL is a multi-disciplinary open access archive for the deposit and dissemination of scientific research documents, whether they are published or not. The documents may come from teaching and research institutions in France or abroad, or from public or private research centers.
L'archive ouverte pluridisciplinaire HAL, est destinée au dépôt et à la diffusion de documents scientifiques de niveau recherche, publiés ou non, émanant des établissements d'enseignement et de recherche français ou étrangers, des laboratoires publics ou privés. 


\title{
Magneto Elastic Modes in Ferro or Ferrimagnetic Polycristals
}

\author{
G. Le Blevennec and I. Brard \\ CEA-CESTA, BP. 2, 33114 Le Barp, France
}

\begin{abstract}
We propose a new method to compute the dimensional variations of ferri or ferro magnetic materials induced by an electromagnetic wave. The algorithm used derives from a mechanical finite element method to solve stress and strain states of a solid. Magnetostriction is written using a quadratic relationship between deformation and magnetization. The results of the numerical computation for nickel samples show the complete frequency spectrum of magneto elastic modes of free standing or clamped sample. Experimental data obtained by measuring frequency dispersion permeabilities shows the coupling rays which are within $3.5 \%$ agreement with the computed frequencies of magneto elastic modes .
\end{abstract}

\section{INTRODUCTION}

The coupling of electromagnetic waves and magneto-elastic modes is at the origin of different phenomena, such as vibrations, noises or mechanical losses in magnetic circuits[1]. Recently numerical approaches [2] have been developed, allowing the computation of more complex situations, specially from the point of view of the boundary conditions occurring in magnetostatic deformation. Besides, experimental techniques have been improved [3] to detect such modes during dynamic excitations.

Here, we propose a numerical method, using finite elements, which enables the computation of the broad band spectrum of magneto elastic modes of a ferri or ferromagnetic material. In what follows, one can replace ferromagnetics by ferrimagnetics without having anything to change in the formalism. In fact, for ferro or ferri isotropic polycrystals, the theoretical descriptions of the saturation magnetostriction are the same [4,5]. The rotation mechanisms of magnetization behave in the same way for both type of materials, so our theory can be applied as well for ferrimagnetics. The chosen algorithm is fairly classical [6] in mechanics. The Joule magnetostriction is introduced by means of a quadratic relationship between relative deformation and magnetization. Further, an experimental validation is realized by measuring the permeability spectra which clearly exhibit sharp peaks resulting from the coupling between electromagnetic waves and deformation modes.

\section{MECHANICAL PROBLEM}

The continuous medium is divided into volume elements linked by a finite number of nodal points where fictive forces originating from the stress distribution are applied. From the stress deformation relationship for each element, it is possible to deduce the properties of the medium and to analyse its behaviour. The algorithm proceeds in two steps. First, the static computation of the equilibrium equations of the forces and the constraints existing at the boundaries are implemented. Second, the dynamic aspect are introduced in the previous equations by including inertial and frictional forces. The solution to this linear elastic problem is enclosed in the principle of minimum energy. The computation considers magnetostriction as an initial deformation of the element, then verifies all the equilibrium and boundary conditions in the element and between elements, in order to evaluate the real displacement of a node structure. Intrinsic material properties needed are density, Young's modulus and Poisson coefficient. The time dependence of the magnetostriction deformation is expressed as an initial deformation in a Dirac field excitation. The result of the computation is the displacement of a node of the structure versus time. Applying the Fourier transform finally gives the broad band spectrum of the mechanical modes. 


\section{NUMERICAL SIMULATION}

The Joule magnetostriction is considered in this work just like any other common initial deformation such as thermal expansion, shrinkage etc... For polycrystals there is no exact relationship between relative magnetostriction and magnetization. Like other authors [7] we use a quadratic approximation to describe a near saturation state expressed as

$$
\frac{\delta \overrightarrow{\mathrm{i}}}{1}=\lambda_{\mathrm{s}}\left(\overrightarrow{\mathrm{M}}_{\mathbf{f}}^{2}-\overrightarrow{\mathrm{M}}_{\mathbf{i}}^{2}\right)=-2 \lambda_{\mathrm{s}} \mathrm{M}_{\mathrm{s}} \delta \overrightarrow{\mathrm{M}}
$$

Practically we consider a disk shaped thin layer of a saturated material along the $x$ direction. The disk is set in the $x y$ plane. $M_{f}=M_{s}-d M$ is the final state, $M_{i}=M_{S}$ is the initial state of magnetization, with $M i$ along the $x$ direction. $M_{s}$ is the saturation magnetization. In weak signal approximation, $\delta M$ is proportional to the rotation of magnetization, it is also proportional to the rotation susceptibility of the material and hence to the amplitude of the applied field. The magnetostriction deformation introduced in the computation satisfies the volume conservation. In these expressions it is worth noting that the deformation is more than merely related to the coefficient of magnetostriction since it is also the produce of three fundamental characteristics of the material, namely, the saturation magnetization, susceptibility, and magnetostriction coefficient. The time dependence introduced in the calculation can be a Dirac or a harmonic function, depending on the finality of the investigation.

\section{RESULTS}

The structure is a cylindrical sample of pure nickel $(\phi 18 \mathrm{~mm}$, thickness $: 0.1 \mathrm{~mm})$ which can be either free standing or clamped to a substrate. The sample is meshed into 180 elements. What comes out first is the y displacement versus time for a selected node at the edge of the sample. In order to extract the excited magneto-elastic frequencies we just apply a Fourier transform. A discrete line spectrum of magneto elastic modes in the range of 0.1 to $1 \mathrm{MHz}$ is thus obtained. An experimental validation is realized by measuring the permeability of the same sample using a coil perturbation method and an impedance meter. The magnetic field is a harmonic field sweeping frequencies from $100 \mathrm{~Hz}$ to $1 \mathrm{MHz}$.

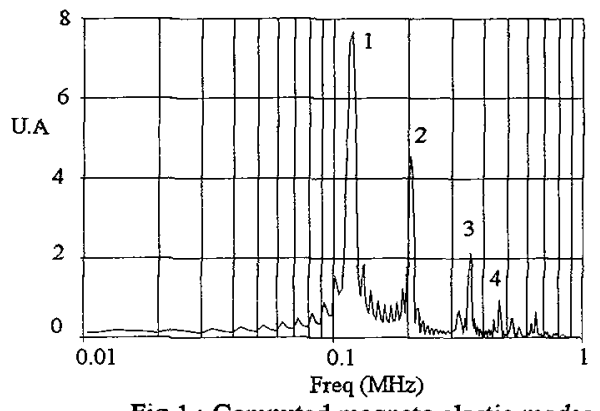

Fig 1 : Computed magneto elastic modes

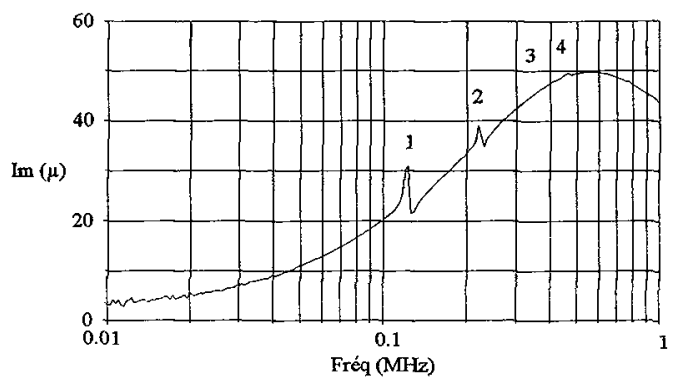

Fig 2 : Experimental imaginary permeability

The magneto elastic modes excited by the harmonic field create a strong perturbation phenomenon which is easily detected on the measured permeability curve (fig 2). The calculation (fig 1) show the same set of 4 main computed lines with an accuracy of $3.5 \%$ with respect to the frequency values. A finer frequency sweeping allows to index each peak, even peak three which is not detectable on the broad spectrum. The fine sweeping shows as the calculation that the relative ray amplitudes decrease with frequency. This coupling which behaves like a perturbation phenomena does not contribute to the magnetization process. In conclusion, the experimental results validate our theoretical approach, thereby providing an efficient tool to investigate more complex structures.

\section{References}

[1] G.W Pierce, Magnetostriction oscillators, Inst of Radio Eng, vol 17,1 (1929).

[2] F. Delince, A Genon, J.M Gillard, H Hedia, W Legros, and A Nicolet, J Appl Phys 69 (8), 15/04/1991.

[3]. D.M Dagenais, F Bucholtz, K.P Koo, and S Vohra, Appl Phys Lett 58 (5),1991.

[4]. E Du Trémolet De Lacheisserie, Magnetostriction, Theory and applications of magnetoelasticity, CRC Press, 1993.

[5]. J Smit and H.P.J Wijn, Les ferrites, Bibliotheque Techniques Philips, 1961.

[6] O.C Zienkiewicz, La methode des elements finis, Mac Graw Hill, 1979.

[7] A.P Thomas, M.R.J Gibbs, J Magn Mat, 103, 1992, p 97-110. 